TRANSACTIONS OF THE

AMERICAN MATHEMATICAL SOCIETY

Volume 357, Number 9, Pages 3673-3682

S 0002-9947(04)03653-0

Article electronically published on December 28, 2004

\title{
DEGENERATION OF LINEAR SYSTEMS THROUGH FAT POINTS ON $K 3$ SURFACES
}

\author{
CINDY DE VOLDER AND ANTONIO LAFACE
}

\begin{abstract}
In this paper we introduce a technique to degenerate $K 3$ surfaces and linear systems through fat points in general position on $K 3$ surfaces. Using this degeneration we show that on generic $K 3$ surfaces it is enough to prove that linear systems with one fat point are non-special in order to obtain the non-speciality of homogeneous linear systems through $n=4^{u} 9^{w}$ fat points in general position. Moreover, we use this degeneration to obtain a result for homogeneous linear systems through $n=4^{u} 9^{w}$ fat points in general position on a general quartic surface in $\mathbb{P}^{3}$.
\end{abstract}

\section{INTRODUCTION}

In this paper we assume the ground field is the field of the complex numbers.

Let $S$ be a smooth projective generic $K 3$ surface (i.e. Pic $(S) \cong \mathbb{Z}$ ) and let $H$ be the generator of Pic $(S)$.

Consider $n$ points in general position on $S$, to each one of them associate a natural number $m_{i}$ called the multiplicity of the point and let $n_{j}$ be the number of points with multiplicity $m_{j}$.

For a linear system of curves in $|d H|$ with $n_{j}$ general base points of multiplicity $m_{j}$ for $j=1, \ldots, k$, define its virtual dimension $v$ as $\operatorname{dim}|d H|-\sum n_{i} m_{i}\left(m_{i}+1\right) / 2$ and its expected dimension by $e=\max \{v,-1\}$. If the dimension of the linear system is $l$, then $v \leq e \leq l$.

Observe that it is possible to have $e<l$, since the conditions imposed by the points may be dependent. In this case we say that the system is special.

Linear systems through general fat points on rational surfaces have been studied by many authors (see e.g. [AH00, [BZ03, CM98, CM01 Eva99]), but, as far as we know, on $K 3$ surfaces, no results on the non-speciality of such systems are known.

In Section 3 we develop a technique to degenerate a $K 3$ surface and linear systems through fat points on $K 3$ surfaces, this degeneration is based on a degeneration of the projective plane which was developed by C. Ciliberto and R. Miranda and used by A. Buckley and M. Zompatori in [BZ03].

Received by the editors October 24, 2003 and, in revised form, January 14, 2004.

2000 Mathematics Subject Classification. Primary 14C20, 14J28.

Key words and phrases. Linear systems, fat points, generic $K 3$ surfaces.

The first author is a Postdoctoral Fellow of the Fund for Scientific Research-Flanders (Belgium) (F.W.O.-Vlaanderen).

The second author would like to thank the European Research and Training Network EAGER for the support provided at Ghent University. He also acknowledges the support of the MIUR of the Italian Government in the framework of the National Research Project "Geometry in Algebraic Varieties" (Cofin 2002). 
In the subsequent section, we use this degeneration to prove that, a homogeneous linear system of curves in $|d H|$ with $n$ general base points of multiplicity $m$ is nonspecial if all linear systems of curves in $|d H|$ with one multiple base point are non-special.

Finally, in Section 5, we prove conjecture [DL03, Conjecture 2.3 (i)] for homogeneous linear systems of curves in $|d H|$ with $4^{u} 9^{w}$ fat points of multiplicity $m$, if either $v \geq 0$ or $v \leq-1$ and $u>0$ or $2 d \neq 1 \bmod 3$.

\section{Preliminaries}

Let $S$ be a smooth projective generic $K 3$ surface and let $H$ be the generator of $\operatorname{Pic}(S)$. Then $H$ is ample, $H^{2}=2 g-2 \geq 2$ and $h^{0}(H)=g+1$; moreover, $H$ is very ample if $g \geq 3$ and if $g=2, H$ defines a double covering of $\mathbb{P}^{2}$ branched at an irreducible sextic (see [May72, Proposition 3]).

Consider $Q_{1}, \cdots, Q_{n}$ points in general position on $S$, for each one of these points fix a multiplicity $m_{1}, \ldots, m_{n}$. Define $\mathcal{L}=\mathcal{L}^{\gamma}\left(d, m_{1}, \ldots, m_{n}\right)$, with $\gamma=H^{2}=2 g-2$, as the linear system of curves in $|d H|$ with multiplicity at least $m_{i}$ at $Q_{i}$ for all $i$. By abuse of notation, let $\mathcal{L}^{\gamma}\left(d, m_{1}, \ldots, m_{n}\right)$ also denote the associated sheaf on $S$. Let $v$ denote the virtual dimension of $\mathcal{L}$. Then, using $\operatorname{dim}|d H|=\frac{\gamma d^{2}}{2}+1$, we obtain that

$$
v=\frac{\gamma d^{2}}{2}+1-\sum_{i=1}^{n} \frac{m_{i}\left(m_{i}+1\right)}{2} .
$$

Let $S^{\prime}$ be the blowing-up of $S$ along the points $Q_{1}, \ldots, Q_{n}$, let $\pi: S^{\prime} \rightarrow S$ be the projection map, and let $E_{i}$ be the exceptional divisor corresponding to $Q_{i}$. The linear system $\mathcal{L}$ then corresponds to the system associated to the line bundle $\pi^{*}\left(\mathcal{O}_{S}(d H)\right) \otimes \mathcal{O}_{S^{\prime}}\left(-m_{1} E_{1}-\cdots-m_{n} E_{n}\right)$. By abuse of notation, we will denote this linear system and its associated line bundle on the blowing-up also by $\mathcal{L}^{\gamma}\left(d, m_{1}, \ldots, m_{n}\right)$.

Similary, by $\mathcal{L}^{\gamma}\left(d, m_{1}^{n_{1}}, \ldots, m_{y}^{n_{y}}\right)$, we denote the linear system of curves in $|d H|$ with $n_{i}$ points (in general position) of multiplicity at least $m_{i}$ (for all $i=1, \ldots, y$ ) as well as the associated sheaf on $S$ and the corresponding linear system and its associated line bundle on the blowing-up of $S$ along those $n_{1}+\cdots+n_{y}$ general points.

Let $Z=\sum_{i=1}^{n} m_{i} Q_{i}$ be the 0 -dimensional scheme of $S$ defined by the multiple points and consider the exact sequence of sheaves:

$$
0 \longrightarrow \mathcal{O}_{S}(d H) \otimes \mathcal{I}_{Z} \longrightarrow \mathcal{O}_{S}(d H) \longrightarrow \mathcal{O}_{Z} \longrightarrow 0
$$

where $\mathcal{I}_{Z}$ is the ideal sheaf of $Z$. Taking cohomology we obtain

$$
v=h^{0}\left(\mathcal{O}_{S}(d H) \otimes \mathcal{I}(Z)\right)-h^{1}\left(\mathcal{O}_{S}(d H) \otimes \mathcal{I}(Z)\right)-1,
$$

because $h^{1}\left(\mathcal{O}_{S}(d H)\right)=0$ (see e.g. May72).

Let $e$ denote the expected dimension of the linear system $\mathcal{L}$, i.e. $e=\max \{-1, v\}$, and let $l$ denote the dimension of $\mathcal{L}$. Then obviously we have that $v \leq e \leq l$.

If $e<l$ (resp. $e=l$ ), we say the linear system $\mathcal{L}$ is special (resp. non-special). Note that equation (2.b) shows that a non-empty linear system $\mathcal{L}$ is non-special if and only if $h^{1}(\mathcal{L})=0$.

Analogously, on $\mathbb{P}^{2}$, consider $n$ general points $Q_{1}, \ldots, Q_{n}$. Let $\mathcal{L}\left(d, m_{1}, \ldots, m_{n}\right)$ denote the linear system of plane curves of degree $d$, having multiplicity at least $m_{i}$ at $Q_{i}$ for all $i=1, \ldots, n$. And let $\mathcal{L}\left(d, m_{1}^{n_{1}}, \ldots, m_{y}^{n_{y}}\right)$ denote the linear system 
of plane curves of degree $d$ having $n_{i}$ points (in general position) of multiplicity at least $m_{i}$ (for all $i=1, \ldots, y$ ). By abuse of notation, we also denote the associated sheafs on $\mathbb{P}^{2}$ and the corresponding linear systems and their associated line bundles on the blowings-up of $\mathbb{P}^{2}$ by $\mathcal{L}\left(d, m_{1}, \ldots, m_{n}\right)$ and $\mathcal{L}\left(d, m_{1}^{n_{1}}, \ldots, m_{y}^{n_{y}}\right)$.

\section{Degeneration of Linear systems on $K 3$ surfaces}

3.A. The degeneration of a $K 3$ surface. Let $S$ be a $K 3$ surface and $\Delta$ a complex disc around the origin. Consider the product $V=S \times \Delta$ and its two projections $q_{1}: V \rightarrow \Delta$ and $q_{2}: V \rightarrow S$. Let $V_{t}$ denote $S \times\{t\}$. Consider $b$ general points in $V_{0}$ and blow up $V$ along those $b$ points. We then get a new threefold $X$ and the maps $\pi: X \rightarrow V, p_{1}=q_{1} \circ \pi: X \rightarrow \Delta$ and $p_{2}=q_{2} \circ \pi: X \rightarrow S$; i.e. we obtain the following commutative diagram:

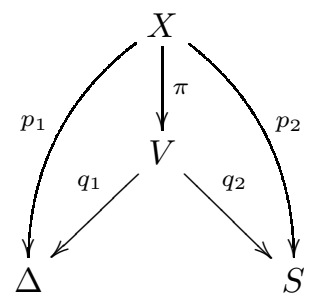

Let $X_{t}$ be the fiber of $p_{1}$ over $t \in \Delta$. If $t \neq 0$, then $X_{t} \cong V_{t}$ is our $K 3$ surface $S$. But, $X_{0}$ is the union of the proper transform $\widetilde{S}$ of $V_{0}$ and the $b$ exceptional divisors $\mathbf{P}_{i}$. Obviously each $\mathbf{P}_{i}$ is isomorphic to $\mathbb{P}^{2}$ and $\widetilde{S}$ is the blowing-up of our $K 3$ surface $S$ at the $b$ general points with projection map $\mathfrak{b}: \widetilde{S} \rightarrow S$.

Every $\mathbf{P}_{i}$ intersects $\widetilde{S}$ transversally along a curve $R_{i}$, which is a line in $\mathbf{P}_{i}$ and an exceptional divisor on $\widetilde{S}$. When we want to indicate that we consider $R_{i}$ in $\widetilde{S}$, resp. $\mathbf{P}_{i}$, we denote it by $E_{i}$, resp. $L_{i}$.

Note that the map $p_{1}$ gives a flat family of surfaces over $\Delta$, so $X_{0}$ can be seen as a degeneration of $S$.

3.B. The degeneration of a linear system on a $K 3$ surface $S$. Let $\mathcal{L}$ be a line bundle on $S$, and, for $k \in \mathbb{Z}$, define the line bundle $\mathcal{O}_{X}(\mathcal{L}, k)$ on $X$ by

$$
\mathcal{O}_{X}(\mathcal{L}, k)=p_{2}^{*}(\mathcal{L}) \otimes \mathcal{O}_{X}(k \widetilde{S})
$$

The restriction of $\mathcal{O}_{X}(\mathcal{L}, k)$ to $X_{t}$, for $t \neq 0$, is then isomorphic to $\mathcal{L}$.

The restriction of $\mathcal{O}_{X}(\mathcal{L}, k)$ to $X_{0}$, which we denote by $\mathcal{X}(\mathcal{L}, k)$, is a flat limit of the line bundle $\mathcal{L}$ on the general fiber $X_{t}$, so $\mathcal{X}(\mathcal{L}, k)$ can be seen as a degeneration of the line bundle $\mathcal{L}$.

On any $\mathbf{P}_{i}$, we have that $\left.\mathcal{X}(\mathcal{L}, k)\right|_{\mathbf{P}_{i}}=\left.\mathcal{O}_{X}(\mathcal{L}, k)\right|_{\mathbf{P}_{i}} \cong \mathcal{O}_{\mathbb{P}^{2}}(k)$, since $\widetilde{S}$ intersects $\mathbf{P}_{i}$ along a line.

On $\widetilde{S}$ we obtain that

$$
\left.\mathcal{X}(\mathcal{L}, k)\right|_{\widetilde{S}}=\left.\mathcal{O}_{X}(\mathcal{L}, k)\right|_{\widetilde{S}}=\left.\left.p_{2}^{*}(\mathcal{L})\right|_{\widetilde{S}} \otimes \mathcal{O}_{X}(k \widetilde{S})\right|_{\widetilde{S}}
$$

But, since $\widetilde{S} \sim X_{t}-\sum_{i=1}^{l} \mathbf{P}_{i}$ as divisors on $X$ and $\mathbf{P}_{i}$ intersects $\widetilde{S}$ along $R_{i}$, this means that

$$
\left.\mathcal{X}(\mathcal{L}, k)\right|_{\widetilde{S}} \cong \mathfrak{b}^{*}(\mathcal{L}) \otimes \mathcal{O}_{\widetilde{S}}\left(-\sum_{i=1}^{b} k E_{i}\right) .
$$


Let $Q_{1}, \ldots, Q_{n}$ be general points on $S$, consider a zero-dimensional subscheme $Z=m_{1} Q_{1}+\cdots+m_{n} Q_{n}$ and let $\mathcal{M}$ be the sheaf $\mathcal{L} \otimes \mathcal{I}_{Z}$, where $\mathcal{I}_{Z}$ denotes the ideal sheaf that defines $Z$. Choose positive integers $a_{1}, \ldots, a_{b}$ such that $a_{1}+\cdots+$ $a_{b} \leq n$. Now, for all $i \in\{1, \ldots, b\}$, consider $a_{i}$ general points on $\mathbf{P}_{i}$; and take $n-\sum_{i=1}^{b} a_{i}$ general points on $\widetilde{S}$. Denote those $n$ points (on the $\mathbf{P}_{i}$ and $\widetilde{S}$ ) by $Q_{i}^{\prime}$ (any order will do), and let $Z^{\prime}$ be the zero-dimensional subscheme of $X_{0}$ given by $m_{1} Q_{1}^{\prime}+\cdots+m_{n} Q_{n}^{\prime}$.

Then we obtain that $\mathcal{X}(\mathcal{L}, k) \otimes \mathcal{I}_{Z^{\prime}}$ is a degeneration of $\mathcal{M}$.

3.C. Homogeneous linear systems on generic $K 3$ surfaces. Let $S$ be a generic $K 3$ surface and consider a homogeneous linear system $\mathcal{L}=\mathcal{L}^{\gamma}\left(d, m^{n}\right)$ on $S$. By abuse of notation, $\mathcal{L}$ also denotes the corresponding sheaf.

Choose positive integers $b$ and $a$ such that $a b \leq n$, let $X$ and $\mathcal{X}\left(\mathcal{O}_{S}(d H), k\right)$ be as constructed before. For all $1 \leq i \leq b$ and $1 \leq j \leq a$, let $Q_{i, j}^{\prime}$ be a general point on $\mathbf{P}_{i}$ and for $1 \leq j \leq n-a b$ let $Q_{i}^{\prime}$ be a general point on $\widetilde{S}$. Now consider the zero-dimensional subscheme

$$
Z^{\prime}=\sum_{i=1}^{n-a b} m Q_{i}^{\prime}+\sum_{\substack{i=1, \ldots, b \\ j=1, \ldots, a}} m Q_{i, j}^{\prime},
$$

On $X_{0}$, resp. $\mathbf{P}_{i}$ and $\widetilde{S}$, define the sheaf $\mathcal{L}_{0}:=\mathcal{X}\left(\mathcal{O}_{S}(d H), k\right) \otimes \mathcal{I}_{Z^{\prime}}$, resp. $\mathcal{L}_{i}:=$ $\left.\mathcal{L}_{0}\right|_{\mathbf{P}_{i}}$ and $\mathcal{L}_{\widetilde{S}}:=\left.\mathcal{L}_{0}\right|_{\widetilde{S}}$. By abuse of notation, we also denote the corresponding linear systems on resp. $X_{0}, \mathbf{P}_{i}$ and $\widetilde{S}$, by resp. $\mathcal{L}_{0}, \mathcal{L}_{i}$ and $\mathcal{L}_{\widetilde{S}}$. Then we see that a divisor in the linear system $\mathcal{L}_{0}$ on $X_{0}$ consists of a divisor $D_{\widetilde{S}} \in \mathcal{L}_{\widetilde{S}}$ and divisors $D_{i} \in \mathcal{L}_{i}$ such that $\left.D_{\widetilde{S}}\right|_{R_{i}}=\left.D_{i}\right|_{R_{i}}$ for all $i$.

By $\mathcal{R}_{\widetilde{S}}$, resp. $\mathcal{R}_{i}$, we denote the linear system on $\bigcup_{i=1}^{b} E_{i}$, resp. $L_{i}$, induced by $\mathcal{L}_{\widetilde{S}}$, resp. $\mathcal{L}_{i}$.

Define the sheafs $\widehat{\mathcal{L}}_{\widetilde{S}}=\mathcal{L}_{\widetilde{S}} \otimes \mathcal{O}_{\widetilde{S}}\left(-\sum_{i=1}^{b} E_{i}\right)$ and $\widehat{\mathcal{L}}_{i}=\mathcal{L}_{i} \otimes \mathcal{O}_{\mathbf{P}_{i}}\left(-L_{i}\right)$, and, again by abuse of notation, let $\widehat{\mathcal{L}}_{\widetilde{S}}$, resp. $\widehat{\mathcal{L}}_{i}$, also denote the corresponding linear system on $\widetilde{S}$, resp. $\mathbf{P}_{i}$.

Notation 3.1.

$$
\begin{aligned}
& l=\operatorname{dim} \mathcal{L}, l_{0}=\operatorname{dim} \mathcal{L}_{0}, \\
& l_{\widetilde{S}}=\operatorname{dim} \mathcal{L}_{\widetilde{S}}, l_{\mathbf{P}}=\operatorname{dim} \mathcal{L}_{i}, \\
& r_{\widetilde{S}}=\operatorname{dim} \mathcal{R}_{\widetilde{S}}, r_{\mathbf{P}}=\operatorname{dim} \mathcal{R}_{i}, \\
& \hat{l}_{\widetilde{S}}=\operatorname{dim} \widehat{\mathcal{L}}_{\widetilde{S}}, \hat{l}_{\mathbf{P}}=\operatorname{dim} \widehat{\mathcal{L}}_{i} .
\end{aligned}
$$

Obviously we have the following:

$$
\begin{aligned}
& l_{\widetilde{S}}=r_{\widetilde{S}}+\hat{l}_{\widetilde{S}}+1, \\
& l_{\mathbf{P}}=r_{\mathbf{P}}+\hat{l}_{\mathbf{P}}+1 .
\end{aligned}
$$

Let $\mathcal{R}_{\cup L_{i}}$ denote the linear system on $\bigcup_{i=1}^{b} R_{i}$ which consists of $\mathcal{R}_{j}$ on $R_{j}$ for all $j=1, \ldots, b$, and denote $r_{\cup L_{i}}=\operatorname{dim} \mathcal{R}_{\cup L_{i}}$. Then we have the following equality:

$$
l_{0}=\operatorname{dim}\left(\mathcal{R}_{\widetilde{S}} \cap \mathcal{R}_{\cup L_{i}}\right)+b\left(\hat{l}_{\mathbf{P}}+1\right)+\hat{l}_{\widetilde{S}}+1 .
$$


But, proceeding as in [BZ03, $\S 2]$, we obtain the transversality of $\mathcal{R}_{\widetilde{S}}$ and $\mathcal{R}_{\cup L_{i}}$; i.e.

$$
\begin{aligned}
\operatorname{dim}\left(\mathcal{R}_{\widetilde{S}} \cap \mathcal{R}_{\cup L_{i}}\right) & =\max \left\{-1, r_{\widetilde{S}}+r_{\cup L_{i}}-b k\right\} \\
& =\max \left\{-1, r_{\widetilde{S}}+b r_{\mathbf{P}}-b k\right\} .
\end{aligned}
$$

For the virtual dimensions of the systems we introduce the following.

Notation 3.2.

$$
\begin{aligned}
& v=\operatorname{vdim} \mathcal{L}, \\
& v_{\mathbf{P}}=\operatorname{vdim} \mathcal{L}_{i}, v_{\widetilde{S}}=\operatorname{vdim} \mathcal{L}_{\widetilde{S}}, \\
& \hat{v}_{\widetilde{S}}=\operatorname{vdim} \widehat{\mathcal{L}}_{\widetilde{S}}, \hat{v}_{\mathbf{P}}=\operatorname{vdim} \widehat{\mathcal{L}}_{i} .
\end{aligned}
$$

Since $\mathcal{L}=\mathcal{L}^{\gamma}\left(d, m^{n}\right), \mathcal{L}_{\widetilde{S}} \cong \mathcal{L}^{\gamma}\left(d, k^{b}, m^{n-a b}\right), \widehat{\mathcal{L}}_{\widetilde{S}} \cong \mathcal{L}^{\gamma}\left(d,(k+1)^{b}, m^{n-a b}\right)$, $\mathcal{L}_{i} \cong \mathcal{L}\left(k, m^{a}\right)$ and $\widehat{\mathcal{L}}_{i} \cong \mathcal{L}\left(k-1, m^{a}\right)$, a simple calculation shows that

$$
\begin{aligned}
v & =v_{\widetilde{S}}+b \hat{v}_{\mathbf{P}}+b=v_{\widetilde{S}}+b\left(v_{\mathbf{P}}-k\right) \\
& =\hat{v}_{\widetilde{S}}+b v_{\mathbf{P}}+b=\hat{v}_{\widetilde{S}}+b\left(\hat{v}_{\mathbf{P}}+k+2\right) .
\end{aligned}
$$

3.D. Remark. Let $S$ be a $K 3$ surface, $\mathcal{L}$ a line bundle on $S$ and let $X$ and $\mathcal{X}(\mathcal{L}, k)$ be as before.

Now do the construction of $\$ 3 . \mathrm{A}$ and $93 . \mathrm{B}$ using $X_{0}$ instead of $S$ and $\mathcal{X}(\mathcal{L}, k)$ $\left(\right.$ or $\left.\mathcal{X}(\mathcal{L}, k) \otimes \mathcal{I}_{Z^{\prime}}\right)$ instead of $\mathcal{L}\left(\right.$ or $\left.\mathcal{L} \otimes \mathcal{I}_{Z}\right)$; i.e. consider $W=X_{0} \times \Delta$, blow up $W$ along $b^{\prime}$ general points on $\widetilde{S} \times\{0\} \subset W_{0}=X_{0} \times\{0\}$ and obtain the following commutative diagram:

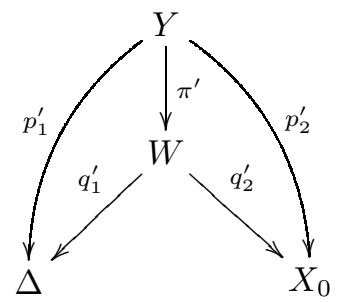

So we obtain a degeneration of $X_{0}$, and a degeneration of $\mathcal{X}(\mathcal{L}, k)($ or $\mathcal{X}(\mathcal{L}, k) \otimes$ $\left.\mathcal{I}_{Z^{\prime}}\right)$.

We call this a double degeneration of $S$ and $\mathcal{L}$ (or $\mathcal{L} \otimes \mathcal{I}_{Z}$ ), and, continuing in the same way, we can obtain an $\eta$-uple degeneration of $S$ and $\mathcal{L}$ (or $\mathcal{L} \otimes \mathcal{I}_{Z}$ ), for any $\eta \geq 2$.

\section{Applying the degeneration to homogeneous linear systems ON GENERIC $K 3$ SURFACES}

From now on we assume that we work on a generic $K 3$ surface $S$, with $H^{2}=\gamma$, where $H$ is the generator of Pic $S$.

The main result of this section is the following.

Theorem 4.1. If $\mathcal{L}^{\gamma}(d, \mu)$ is non-special for all $\mu$, then $\mathcal{L}=\mathcal{L}^{\gamma}\left(d, m^{n}\right)$ with $n=$ $4^{u} 9^{w}$ is non-special for all positive integers $m, u$ and $w$.

To make the proof of this theorem more transparent we first fix some notation and state a few auxiliary results.

Let $c \in\{4,9\}$ such that $c \mid n$ and consider the degeneration $\left(X_{0}, \mathcal{L}_{0}\right)$ of $(S, \mathcal{L})$ obtained by using the construction explained in $\S[$ with $a=c, b=n / c$ and $k \in \mathbb{N}$. Note that, since $\mathcal{L}_{0}$ is a degeneration of $\mathcal{L}, l_{0} \geq l \geq e \geq v$. 
On $\mathbb{P}^{2}$, let $\mathcal{L}\left(\delta, \mu^{\nu}\right)$ denote the linear system of plane curves of degree $\delta$ having multiplicity $\mu$ at $\nu$ points (in general position).

The following is a well-known result, and can easily be checked using, for instance, the results of Har85.

Lemma 4.2. On $\mathbb{P}^{2}$ the linear system $\mathcal{L}\left(\delta, \mu^{c}\right)$ with $c \in\{4,9\}$ is non-special for all $\delta$ and $\mu$.

Remark 4.3. Lemma4.2 implies, in particular, that $\mathcal{L}_{i}$ and $\widehat{\mathcal{L}}_{i}$ are non-special linear systems on $\mathbf{P}_{i}$.

Claim 4.4. If $v \geq-1, v_{\widetilde{S}} \geq-1, v_{\mathbf{P}} \geq-1$ and $\mathcal{L}_{\widetilde{S}}$ and $\widehat{\mathcal{L}}_{\widetilde{S}}$ are non-special systems; then $\operatorname{dim}\left(\mathcal{R}_{\widetilde{S}} \cap \mathcal{R}_{\cup L_{i}}\right)=r_{\widetilde{S}}+b r_{\mathbf{P}}-b k$.

Proof. Note that the conditions of the theorem immediately imply that $l_{\widetilde{S}}=v_{\widetilde{S}}$ and $l_{\mathbf{P}}=v_{\mathbf{P}}$.

Since $\operatorname{dim}\left(\mathcal{R}_{\widetilde{S}} \cap \mathcal{R}_{\cup L_{i}}\right)=\max \left\{-1, r_{\widetilde{S}}+b r_{\mathbf{P}}-b k\right\}$, it suffices to show that $r_{\widetilde{S}}+b r_{\mathbf{P}}-b k \geq-1$.

Because of (3.c) and (3.d), we have that

$$
r_{\widetilde{S}}+b r_{\mathbf{P}}-b k=l_{\widetilde{S}}-\hat{l}_{\widetilde{S}}-1+b\left(l_{\mathbf{P}}-\hat{l}_{\mathbf{P}}-1\right)-b k .
$$

If $\hat{v}_{\widetilde{S}} \geq-1$ and $\hat{v}_{\mathbf{P}} \geq-1$, then $\hat{l}_{\widetilde{S}}=\hat{v}_{\widetilde{S}}$ and $\hat{l}_{\mathbf{P}}=\hat{v}_{\mathbf{P}}$. So

$$
\begin{aligned}
r_{\widetilde{S}}+b r_{\mathbf{P}}-b k & =v_{\widetilde{S}}-\hat{v}_{\widetilde{S}}-1+b\left(v_{\mathbf{P}}-\hat{v}_{\mathbf{P}}-1\right)-b k \\
& =b(k+1)-1 \geq-1 .
\end{aligned}
$$

If $\hat{v}_{\widetilde{S}} \leq-2$ and $\hat{v}_{\mathbf{P}} \geq-1$, then $\hat{l}_{\widetilde{S}}=-1$ and $\hat{l}_{\mathbf{P}}=\hat{v}_{\mathbf{P}}$. So

$$
\begin{aligned}
r_{\widetilde{S}}+b r_{\mathbf{P}}-b k & =v_{\widetilde{S}}+b\left(v_{\mathbf{P}}-\hat{v}_{\mathbf{P}}-1\right)-b k \\
& =v_{\widetilde{S}} \geq-1 .
\end{aligned}
$$

If $\hat{v}_{\widetilde{S}} \geq-1$ and $\hat{v}_{\mathbf{P}} \leq-2$, then $\hat{l}_{\widetilde{S}}=\hat{v}_{\widetilde{S}}$ and $\hat{l}_{\mathbf{P}}=-1$. So

$$
\begin{aligned}
r_{\widetilde{S}}+b r_{\mathbf{P}}-b k & =v_{\widetilde{S}}-\hat{v}_{\widetilde{S}}-1+b v_{\mathbf{P}}-b k \\
& =b\left(1+v_{\mathbf{P}}\right)-1 \geq-1 .
\end{aligned}
$$

If $\hat{v}_{\widetilde{S}} \leq-2$ and $\hat{v}_{\mathbf{P}} \leq-2$, then $\hat{l}_{\widetilde{S}}=-1$ and $\hat{l}_{\mathbf{P}}=-1$. So

$$
\begin{gathered}
r_{\widetilde{S}}+b r_{\mathbf{P}}-b k=v_{\widetilde{S}}+b\left(v_{\mathbf{P}}-k\right) \\
\stackrel{3 . g}{=} v \geq-1 .
\end{gathered}
$$

Lemma 4.5. If $v \geq-1, v_{\widetilde{S}} \geq-1, v_{\mathbf{P}} \geq-1$ and $\mathcal{L}_{\widetilde{S}}$ and $\widehat{\mathcal{L}}_{\widetilde{S}}$ are non-special systems; then $\mathcal{L}$ is non-special.

Proof. Because $\mathcal{L}_{0}$ is a degeneration of $\mathcal{L}$, we know that $v \leq l \leq l_{0}$; so it suffices to prove that $l_{0}=v$.

Using (3.e), (3.f) and Claim 4.4 we obtain that

$$
l_{0}=r_{\widetilde{S}}+b r_{\mathbf{P}}-b k+b\left(\hat{l}_{\mathbf{P}}+1\right)+\hat{l}_{\widetilde{S}}+1 .
$$

So, using (3.c) and (3.d), we see that

$$
l_{0}=l_{\widetilde{S}}+b\left(l_{\mathbf{P}}-k\right)=v_{\widetilde{S}}+b\left(v_{\mathbf{P}}-k\right) \stackrel{\text { 3.g. }}{-} v .
$$


Lemma 4.6. Let $\mathcal{L}=\mathcal{L}^{\gamma}\left(d, m^{n}\right)$ with $n=4^{u} 9^{w}, d, m, u, w \in \mathbb{N}, u+w>0$ and $v \geq-1$. Take $c, b, X_{0}$ and $\mathcal{L}_{0}$ as before. Then $\exists k \in \mathbb{N}$ such that $v_{\widetilde{S}} \geq-1$ and $v_{\mathbf{P}} \geq-1$.

Proof. Because $v_{\widetilde{S}}=\frac{\gamma}{2} d^{2}+1-b \frac{k(k+1)}{2}$ and $v_{\mathbf{P}}=\frac{k(k+3)}{2}-c \frac{m(m+1)}{2}$, the inequalities $v_{\widetilde{S}} \geq-1$ and $v_{\mathbf{P}} \geq-1$ are equivalent to

$$
\begin{aligned}
k^{2}+k-\alpha \leq 0, & \text { with } \alpha=\frac{1}{b}\left(\gamma d^{2}+4\right), \\
k^{2}+3 k-\beta \geq 0, \quad \text { with } \beta & =c m(m+1)-2 .
\end{aligned}
$$

But this is the same as

$$
\begin{aligned}
& k \in\left[\frac{-1-\sqrt{1+4 \alpha}}{2}, \frac{-1+\sqrt{1+4 \alpha}}{2}\right], \\
& \left.k \in]-\infty, \frac{-3-\sqrt{9+4 \beta}}{2}\right] \cup\left[\frac{-3+\sqrt{9+4 \beta}}{2}, \infty[.\right.
\end{aligned}
$$

So proving the statement is equivalent to proving that there exists a positive integer $k$ such that both (4.j) and (4.k) are satisfied, i.e. it is enough to show that

$$
\frac{-3+\sqrt{9+4 \beta}}{2}+1 \leq \frac{-1+\sqrt{1+4 \alpha}}{2} .
$$

A simple calculation shows that the previous inequality is equivalent to $\alpha \geq \beta+2$, which is in turn equivalent to $v \geq-1$.

Lemma 4.7. If $v \leq-1, \hat{v}_{\widetilde{S}} \leq-1, \hat{v}_{\mathbf{P}} \leq-1$ and $\mathcal{L}_{\widetilde{S}}$ and $\widehat{\mathcal{L}}_{\widetilde{S}}$ are non-special systems, then $\mathcal{L}$ is non-special and thus empty.

Proof. Since $v_{\widetilde{S}}=\hat{v}_{\widetilde{S}}+b(k+1)$ and $v_{\mathbf{P}}=\hat{v}_{\mathbf{P}}+k+1$, we see that $v_{\widetilde{S}} \leq b(k+1)-1$ and $v_{\mathbf{P}} \leq k$.

If $v_{\widetilde{S}} \leq-1$ and $v_{\mathbf{P}} \leq-1$, then $r_{\widetilde{S}}=r_{\mathbf{P}}=-1$. If $v_{\widetilde{S}} \leq-1$ and $v_{\mathbf{P}} \geq-1$, then $r_{\widetilde{S}}=-1$ and $r_{\mathbf{P}}=v_{\mathbf{P}} \leq k$. If $v_{\widetilde{S}} \geq-1$ and $v_{\mathbf{P}} \leq-1$, then $r_{\widetilde{S}}=v_{\widetilde{S}} \leq b(k+1)-1$ and $r_{\mathbf{P}}=-1$. If $v_{\widetilde{S}} \geq-1$ and $v_{\mathbf{P}} \leq-1$, then $r_{\widetilde{S}}=v_{\widetilde{S}}$ and $r_{\mathbf{P}}=v_{\mathbf{P}}$. So in any case we obtain that $r_{\widetilde{S}}+b r_{\mathbf{P}}-b k \leq-1$, i.e. $\operatorname{dim}\left(\mathcal{R}_{\widetilde{S}} \cap \mathcal{R}_{\cup L_{i}}\right)=-1$.

Using (3.e) and $\hat{l}_{\widetilde{S}}=\hat{l}_{\mathbf{P}}=-1$, we see that $l_{0}=\hat{l}_{\widetilde{S}}+k\left(\hat{l}_{\mathbf{P}}+1\right)=-1$.

Lemma 4.8. Let $\mathcal{L}=\mathcal{L}^{\gamma}\left(d, m^{n}\right)$ with $n=4^{u} 9^{w}, d, m, u, w \in \mathbb{N}, u+w>0$ and $v \leq-1$. Take $c, b, X_{0}$ and $\mathcal{L}_{0}$ as before. Then $\exists k \in \mathbb{N}$ such that $\hat{v}_{\widetilde{S}} \leq-1$ and $\hat{v}_{\mathbf{P}} \leq-1$.

Proof. Because $\hat{v}_{\widetilde{S}}=\frac{\gamma}{2} d^{2}+1-b \frac{(k+1)(k+2)}{2}$ and $\hat{v}_{\mathbf{P}}=\frac{(k-1)(k+2)}{2}-c \frac{m(m+1)}{2}$, the inequalities $\hat{v}_{\widetilde{S}} \leq-1$ and $\hat{v}_{\mathbf{P}} \leq-1$ are equivalent to

$$
\begin{aligned}
k^{2}+3 k-\alpha \geq 0, \quad \text { with } \alpha & =\frac{1}{b}\left(\gamma d^{2}+4\right)-2, \\
k^{2}+k-\beta \leq 0, \quad \text { with } \beta & =c m(m+1) .
\end{aligned}
$$

Proceeding as in the proof of Lemma 4.6, we obtain that it is sufficient to prove that

$$
\frac{-1+\sqrt{1+4 \beta}}{2} \geq \frac{-3+\sqrt{9+4 \alpha}}{2}+1
$$

which is equivalent to $\beta \geq \alpha+2$. This last inequality is equivalent to $v \geq-1$.

Proof of Theorem 4.1. Using induction, the result follows immediately from Lemmas 4.5 and 4.6 if $v \geq-1$, and from Lemmas 4.7 and 4.8 if $v \leq-1$. 
4.A. Using a higher order degeneration. Using the $\eta$-uple degeneration explained in Remark 3.D we can, proceeding as above, prove that the non-speciality of $\mathcal{L}^{\gamma}\left(d, \mu_{1}, \ldots, \mu_{\eta}\right)$ for all $\mu_{1}, \ldots, \mu_{\eta}$ implies the non-speciality of

$$
\mathcal{L}=\mathcal{L}^{\gamma}\left(d, m_{1}^{n_{1}}, \ldots, m_{\eta}^{n_{\eta}}\right)
$$

with $n_{i}=4^{u_{i}} 9^{w_{i}}$ for all $m_{1}, \ldots, m_{\eta}, u_{1}, \ldots, u_{\eta}, w_{1}, \ldots, w_{\eta}$.

As we will see in Proposition 5.3. proving the non-speciality of systems $\mathcal{L}^{\gamma}(d, \mu)$ is already rather complex. In fact, in Proposition 5.3 we only prove when such a system is non-special for general generic $K 3$ surfaces with $\gamma=4$.

4.B. Remark. As proved in [CM01, Theorem 6.1], the Segre conjecture on planar linear systems through fat points implies that the only special homogeneous linear systems $\mathcal{L}\left(k, m^{n}\right)$ are the ones with $n \in\{2,3,5,6,7,8\}$. So if the Segre conjecture is true, we can do the degeneration using, not only $a=4$ or 9 , but $a \in\{4\} \cup \mathbb{Z}_{\geq 9}$. In this way we can then prove Theorem 4.1, for any $n$ which can be written as a product of powers of numbers in $\{4\} \cup \mathbb{Z}_{\geq 9}$, and thus, in particular, for any $n \geq 9$. Note that the idea of using the results on $\mathbb{P}^{2}$ to reduce the problem of linear systems through fat points to a problem involving a single fat point has previously come up in the closely related context of Seshadri constants (see Roe03, Theorem 3 and Corollary 8])

\section{The NON-SPECIALITY OF LINEAR SYSTEMS $\mathcal{L}^{\gamma}\left(d, m^{n}\right)$ With $n=4^{u} 9^{w}$ ON GENERAL GENERIC $K 3$ SURFACES WITH $\gamma=4$}

The main result of this section is the following.

Theorem 5.1. Let $S$ be a general generic $K 3$ surface with $\gamma=4$ and let $\mathcal{L}=$ $\mathcal{L}^{4}\left(d, m^{n}\right)$, with $n=4^{u} 9^{w}, u, w \in \mathbb{Z}_{\geq 0}$ and $d \in \mathbb{Z}_{>0}$.

(1) If $v(\mathcal{L}) \geq 0$, then the linear system $\mathcal{L}$ is non-special.

(2) If $v(\mathcal{L}) \leq-1$ and either $u>0$ or $2 d \neq 1 \bmod 3$, then $\mathcal{L}$ is non-special unless $n=1, m=2 d$ and $d \geq 2$. In the latter case $\operatorname{dim}\left(\mathcal{L}^{4}(d, 2 d)\right)=0>$ $\operatorname{edim}\left(\mathcal{L}^{4}(d, 2 d)\right)=-1$ and $\mathcal{L}^{4}(d, 2 d)=d C$, with $C$ the unique element of $\mathcal{L}^{4}(1,2)$.

Remark 5.2. Note that the previous theorem implies that [DL03. Conjecture 2.3 (i)] is true for homogeneous linear systems with $n=4^{u} 9^{w}$ fat points on $S$ in the following cases:

(i) $v \geq 0$, or

(ii) $v \leq-1$ and $u>0$, or

(iii) $v \leq-1$ and $2 d \neq 1 \bmod 3$.

We will prove Theorem 5.1 by using the degeneration as introduced in $\$ 4$ and the following.

Proposition 5.3. Let $S$ be a general generic K3 surface with $\gamma=4$. Then $\mathcal{L}=$ $\mathcal{L}^{4}(d, \mu)$, with $d, \mu \in \mathbb{Z}_{>0}$, is non-special unless $\mu=2 d$ and $d \geq 2$. In the latter case $\operatorname{dim}\left(\mathcal{L}^{4}(d, 2 d)\right)=0>\operatorname{vdim}\left(\mathcal{L}^{4}(d, 2 d)\right)=-1$ and $\mathcal{L}^{4}(d, 2 d)=d C$, with $C$ the unique element of $\mathcal{L}^{4}(1,2)$.

Proof. The map $\phi: S \rightarrow \mathbb{P}^{3}$ corresponding to $|H|$ (with $H$ the generator of Pic $S$ ) is an embedding, so we may look at $S$ as a quartic surface in $\mathbb{P}^{3}$. The unique element $C$ of $\mathcal{L}^{4}(1,2)$ is then the divisor on $S \subset \mathbb{P}^{3}$ defined by the tangent plane to $S$ at 
this general point $P$. So $C$ is an irreducible plane curve of degree 4 having a node at $P$ (and no other singularities).

Blow up $S$ along the point $P$, and recall that, by abuse of notation, $\mathcal{L}^{4}(d, \mu)$ also denotes the complete linear system and the line bundle on this blowing-up corresponding to $\mathcal{L}^{4}(d, \mu)$ on $S$. Let $\widetilde{C}$ denote the strict transform of $C$ on this blowing-up; then $g(\widetilde{C})=2$.

Assume $\mu=2 d(d \geq 2)$ and consider the following sequence:

$$
0 \longrightarrow \mathcal{L}^{4}(d-1,2 d-2) \longrightarrow \mathcal{L}^{4}(d, 2 d) \longrightarrow \mathcal{L}^{4}(d, 2 d) \otimes \mathcal{O}_{\widetilde{C}} \longrightarrow 0
$$

Because $\operatorname{deg}\left(\mathcal{L}^{4}(d, 2 d) \otimes \mathcal{O}_{\widetilde{C}}\right)=0=2 g(\widetilde{C})-4$, we know that $h^{0}\left(\mathcal{L}^{4}(d, 2 d) \otimes \mathcal{O}_{\widetilde{C}}\right)=0$ unless $\mathcal{L}^{4}(d, 2 d) \otimes \mathcal{O}_{\widetilde{C}}=\mathcal{O}_{\widetilde{C}}$. But $\mathcal{L}^{4}(d, 2 d) \otimes \mathcal{O}_{\widetilde{C}}=\left|d g_{4}^{2}-2 d\left(P_{1}+P_{2}\right)\right|$, where $P_{1}$ and $P_{2}$ are the intersection points of $\widetilde{C}$ with the exceptional curve. So this would mean that $\left|d g_{4}^{2}-2 d\left(P_{1}+P_{2}\right)\right|=\mathcal{O}_{\widetilde{C}}$. But, since $P$ is a general point on the general quaritc $S$, we know that $g_{4}^{2}=\left|K_{\widetilde{C}}+P_{1}+P_{2}\right|$; so we would obtain that $\left|d K_{\widetilde{C}}-d\left(P_{1}+P_{2}\right)\right|=\mathcal{O}_{\widetilde{C}}$, or thus that $\left|d K_{\widetilde{C}}\right|=\left|d\left(P_{1}+P_{2}\right)\right|$, which is not true (because $P$ is a general element of $S)$. So $h^{0}\left(\mathcal{L}^{4}(d, 2 d)\right)=h^{0}\left(\mathcal{L}^{4}(d-1,2(d-1))\right)$ for all $d \geq 2$. Using this a number of times we thus obtain that $h^{0}\left(\mathcal{L}^{4}(d, 2 d)\right)=$ $h^{0}\left(\mathcal{L}^{4}(1,2)\right)=1$, so $d C$ is the only divisor in $\mathcal{L}^{4}(d, 2 d)$.

It now follows immediately that $\operatorname{dim} \mathcal{L}=-1$ if $\mu \geq 2 d+1$, since the only divisor in $\mathcal{L}^{4}(d, 2 d)$ has multiplicity exactly $2 d$ in $P$.

Now consider the case $\mu \leq 2 d-1$. If we can prove that $\mathcal{L}$ is non-special for $\mu=2 d-1$, then the non-speciality follows for all $\mu \leq 2 d-1$ (since $\operatorname{vdim} \mathcal{L}^{4}(d, 2 d-1)$ $=d+1)$. For $d=1$, there is nothing to prove, since $P$ is a general point of $S$. So we may assume that $d \geq 2$ and that the $\mathcal{L}^{4}\left(d^{\prime}, 2 d^{\prime}-1\right)$ is non-special for $d^{\prime} \leq d-1$. Now consider the following sequence:

$$
0 \longrightarrow \mathcal{L}^{4}(d-1,2 d-3) \longrightarrow \mathcal{L}^{4}(d, 2 d-1) \longrightarrow \mathcal{L}^{4}(d, 2 d-1) \otimes \mathcal{O}_{\widetilde{C}} \longrightarrow 0 .
$$

Because

$$
\operatorname{deg}\left(\mathcal{L}^{4}(d, 2 d-1) \otimes \mathcal{O}_{\widetilde{C}}\right)=2=2 g(\widetilde{C})-2,
$$

we know that $h^{1}\left(\mathcal{L}^{4}(d, 2 d-1) \otimes \mathcal{O}_{\widetilde{C}}\right)=0$ unless $\mathcal{L}^{4}(d, 2 d-1) \otimes \mathcal{O}_{\widetilde{C}}=K_{\widetilde{C}}$ (the canonical class on $\widetilde{C})$. But $\mathcal{L}^{4}(d, 2 d) \otimes \mathcal{O}_{\widetilde{C}}=\left|d g_{4}^{2}-(2 d-1)\left(P_{1}+P_{2}\right)\right|$ and $g_{4}^{2}=$ $\left|K_{\widetilde{C}}+P_{1}+P_{2}\right|$ (where $P_{1}$ and $P_{2}$ are, as before, the intersection points of $\widetilde{C}$ with the exceptional curve). Then this would mean that $\left|(d-1) K_{\widetilde{C}}\right|=\left|(d-1)\left(P_{1}+P_{2}\right)\right|$, which is not true (because $P$ is a general element of $S)$. So $h^{1}\left(\mathcal{L}^{4}(d, 2 d-1) \otimes \mathcal{O}_{\widetilde{C}}\right)=0$ and, by hypotheses, $h^{1}\left(\mathcal{L}^{4}(d-1,2 d-3)\right)=0$, thus also $h^{1}\left(\mathcal{L}^{4}(d, 2 d-1)\right)=0$.

Proof of Theorem 5.1. (1) In case $n=1$ we are done because of Proposition 5.3 So we assume $n>1$, or equivalently $u+w \geq 1$. Consider the degeneration $\left(X_{0}, \mathcal{L}_{0}\right)$ of $(S, \mathcal{L})$ obtained by using the construction explained in $\S 3$ with $a=n, b=1$ and $k=2 d-1$. Then we have that $\mathcal{L}_{\widetilde{S}}=\mathcal{L}^{4}(d ; 2 d-1), \widehat{\mathcal{L}}_{\widetilde{S}}=\mathcal{L}^{4}(d ; 2 d), \mathcal{L}_{1}=$ $\mathcal{L}\left(2 d-1 ; m^{n}\right)$ and $\widehat{\mathcal{L}}_{1}=\mathcal{L}\left(2 d-2 ; m^{n}\right)$. So, because of Proposition 5.3 , we know that $l_{\widetilde{S}}=v_{\widetilde{S}}(=d+1)$ and $\hat{l}_{\widetilde{S}}=0\left(>\hat{v}_{\widetilde{S}}\right)$. A simple calculation shows that $v_{\mathbf{P}}=v+d-2 \geq v \geq 0$. Moreover, because of [BZ03, Theorem 4], we know that $\mathcal{L}_{1}$ and $\widehat{\mathcal{L}}_{1}$ are non-special. Proceeding as in the proof of Claim 4.4 , one can easily check (using $v \geq 0$ ) that $\operatorname{dim}\left(\mathcal{R}_{\widetilde{S}} \cap \mathcal{R}_{\cup L_{i}}\right)=r_{\widetilde{S}}+r_{\mathbf{P}}-2 d+1$. So, using (3.e), we obtain that

$$
l_{0}=r_{\widetilde{S}}+r_{\mathbf{P}}-2 d+1+\hat{l}_{\widetilde{S}}+l_{\mathbf{P}}+2=l_{\widetilde{S}}+l_{\mathbf{P}}-2 d+1=v_{\widetilde{S}}+v_{\mathbf{P}}-k=v .
$$


(2) Again, if $n=1$ we are done because of Proposition 5.3. So we assume $n>1$, or equivalently $u+w \geq 1$. As in the proof of Theorem 4.1, we can use Lemmas 4.7 and 4.8, up to the last step. For this last step, we have $c=n$ and $b=1$, and we will take $k=2 d$. In this case, we can no longer use Lemma 4.7 since $\mathcal{L}_{\widetilde{S}}$ is special. If we can prove, however, that $\operatorname{dim}\left(\mathcal{R}_{\widetilde{S}} \cap \mathcal{R}_{\cup L_{i}}\right)=\hat{l}_{\widetilde{S}}=\hat{l}_{\mathbf{P}}=-1$, then we still obtain $l_{0}=-1$, and thus also $l=-1$. Since $\operatorname{dim}\left(\mathcal{R}_{\widetilde{S}} \cap \mathcal{R}_{\cup L_{i}}\right)=\max \left\{-1, r_{\widetilde{S}}+\left(r_{\mathbf{P}}-2 d\right)\right\}$, $r_{\widetilde{S}}=0$ and $r_{\mathbf{P}}=l_{\mathbf{P}}$ it is enough to prove that, for $k=2 d, v_{\mathbf{P}} \leq 2 d-1$ and $\hat{v}_{\mathbf{P}} \leq-1$. These inequalities are equivalent to respectively $v \leq-d$ and $v \leq 1-d$, so it is sufficient to have $v \leq-d$.

In case $u>0$, we can make sure that in the last step $c=4$, and we obtain

$$
v \leq-1 \Longleftrightarrow m \geq d \Longleftrightarrow v \leq 1-2 d \text {. }
$$

In case $u=0$, we have $c=9$ in the last step, and using the fact that $2 d \neq 1 \bmod 3$, a simple calculation shows that

$$
v \leq-1 \Longleftrightarrow m \geq \frac{2 d}{3} \Longleftrightarrow v \leq 1-3 d .
$$

\section{ACKNOWLEDGEMENT}

Both authors would like to thank Marc Coppens for helpful discussions on the subject.

\section{REFERENCES}

[AH00] J. Alexander and A. Hirschowitz. An asymptotic vanishing theorem for generic unions of multiple points. Invent. Math., 140(2):303-325, 2000. MR1756998 (2001i:14024)

[BZ03] Anita Buckley and Marina Zompatori. Linear systems of plane curves with a composite number of base points of equal multiplicity. Trans. Amer. Math. Soc., 355(2):539-549 (electronic), 2003. MR 1932712 (2003j:14039)

[CM98] Ciro Ciliberto and Rick Miranda. Degenerations of planar linear systems. J. Reine Angew. Math., 501:191-220, 1998. MF1637857 (2000m:14005)

[CM01] Ciro Ciliberto and Rick Miranda. The Segre and Harbourne-Hirschowitz conjectures. In Applications of algebraic geometry to coding theory, physics and computation (Eilat, 2001), volume 36 of NATO Sci. Ser. II Math. Phys. Chem., pages 37-51. Kluwer Acad. Publ., Dordrecht, 2001. MR1866890 (2002e:14001)

[DL03] Cindy De Volder and Antonio Laface. Linear systems on generic K3 surfaces. Preprint, math.AG/0309073, 2003.

[Eva99] Laurent Evain. La fonction de Hilbert de la réunion de $4^{h}$ gros points génériques de $\mathbf{P}^{2}$ de même multiplicité. J. Algebraic Geom., 8(4):787-796, 1999. MR1703614 (2000e:13023)

[Gim89] Alessandro Gimigliano. Regularity of linear systems of plane curves. J. Algebra, 124(2):447-460, 1989. MR1011606 (90i:14005)

[Har85] Brian Harbourne. Complete linear systems on rational surfaces. Trans. Amer. Math. Soc., 289(1):213-226, 1985. MR0779061 (86h:14030)

[May72] Alan L. Mayer. Families of K3 surfaces. Nagoya Math. J., 48:1-17, 1972. MR0330172 (48:8510)

[Roe03] Joaquim Roé. On the Nagata conjecture. Preprint, math.AG/0304124, 2003.

Department of Pure Mathematics and Computer Algebra, Ghent University, GalGlaAn 2, B-9000 Ghent, Belgium

E-mail address: cdv@cage.ugent.be

Dipartimento di Matematica, Università degli Studi di Milano, Via Saldini 50, 20100 Milano, Italy

E-mail address: antonio.laface@unimi.it 